\title{
Bacteriological Profile and Antibiogram of Cultures Isolated from Tracheal Secretions
}

Hassaan Ahmad ${ }^{1}$, Abdullah Sadiq ${ }^{2}$, Hamza Waqar Bhatti ${ }^{2}$, Awais A. Bhatti ${ }^{3}$, Ahsan Tameezud-din ${ }^{4}$, Ahmed Ibrahim ${ }^{4}$, Noman A. Chaudhary ${ }^{2}$

1. Miscellaneous, Rawalpindi Medical University, Rawalpindi, PAK 2. Surgery, Rawalpindi Medical University, Rawalpindi, PAK 3. Medicine, Rawalpindi Medical University, Rawalpindi, PAK 4. Internal Medicine, Rawalpindi Medical University, Rawalpindi, PAK

$\square$ Corresponding author: Abdullah Sadiq, abdullahsadiq3947@gmail.com Disclosures can be found in Additional Information at the end of the article

\section{Abstract}

\section{Background}

Respiratory infections are associated with high morbidity and mortality, especially in critically ill patients. The excessive use of broad-spectrum antibiotics has led to the development of drug resistance, thus resulting in the emergence of pathogens which are difficult to treat. The aim of this study was to identify common pathogens in tracheal secretions and to study the patterns of their sensitivity and resistance to various antibiotics.

\section{Materials and methods}

This descriptive cross-sectional study was conducted in the Department of Pathology and Microbiology, Holy Family Hospital, Rawalpindi, Pakistan, from August 2017 to December 2017, using the convenient sampling technique. Tracheal secretions from patients in the intensive care unit (ICU), tested in the Pathology and Microbiology Department of Holy Family Hospital, were included in the study. The culture was done on blood and MacConkey agar and the sensitivity pattern was performed on Muller Hinton agar. Data were analyzed using SPSS v.23.0.

\section{Results}

Out of the bacteria isolated from positive growth cultures, Acinetobacter ( $45 ; 53.6 \%)$ was the most common isolate followed by Klebsiella $(11 ; 13.1 \%)$. Acinetobacter was most sensitive to tigecycline (94.7\%), and gram-negative bacteria such as Acinetobacter, Klebsiella, and Pseudomonas showed resistance to higher generation cephalosporins.

\section{Conclusion}

Received 05/28/2019 Review began 06/10/2019 Review ended 06/13/2019 Published 06/21/2019

\section{(C) Copyright 2019}

Ahmad et al. This is an open access article distributed under the terms of the Creative Commons Attribution License CC-BY 3.0., which permits unrestricted use, distribution, and reproduction in any medium, provided the original author and source are credited.
Acinetobacter was the most common gram-negative bacilli isolated. Tigecycline was found to be effective against Acinetobacter.

Categories: Pathology, Infectious Disease

Keywords: gram-negative bacteria, drug resistance, respiratory infections, tracheal secretions

\section{Introduction}

Tracheobronchial secretions are produced by mucous glands and goblet cells of the tracheobronchial tree [1]. These secretions are not only involved in the protection of the 
respiratory tract but are also responsible for the exchange of heat and water during breathing $[2]$.

Respiratory infections are associated with high morbidity and mortality, especially in critically ill patients [3]. Such patients are usually maintained using invasive devices which themselves tend to be a major reservoir for hospital-acquired infections [4,5]. About 15\% of hospitalacquired infections (HAIs) are caused by ventilator-associated pneumonia (VAP). This is the second-most-common HAI having the highest morbidity and mortality [6].

Moreover, the advent and increase in multi-drug resistant (MDR) pathogens serve as a major problem [7]. The excessive and indiscriminate use of broad-spectrum antibiotics has led to the development of these resilient microbes which are difficult to treat $[8,9]$.

The aim of this study was to identify the common pathogens in tracheal secretions and to study the patterns of their sensitivity and resistance to various antibiotics, which can serve as guidelines to physicians for empirical treatment with proper antibiotics.

\section{Materials And Methods}

This descriptive cross-sectional study was conducted in the Department of Pathology and Microbiology, Holy Family Hospital, Rawalpindi, Pakistan, from August 2017 to December 2017. All patients whose tracheal secretion samples were tested in the Pathology and Microbiology Department of Holy Family Hospital were included in the study. In total, 186 samples were selected, regardless of age and gender, by using the convenient sampling technique. Tracheal secretions from patients admitted in the intensive care unit (ICU) for more than 48 hours were obtained by sterile suctioning using an endotracheal tube and suction catheter tip. Samples were inoculated on agar plates. The culture was done on blood and MacConkey agar and was incubated at $37^{\circ} \mathrm{C}$ for 24 to 48 hours. Microbes were identified under a microscope by observing morphological characteristics after gram staining and applying biochemical tests. Antibiotic sensitivity pattern was done on Muller Hinton agar using the Kirby Bauer disk diffusion method. Antibiotic discs containing amikacin, amoxicillin clavulanate (augmentin), aztreonam, ceftazidime, cefoperazone + sulbactam (sulzone), ciprofloxacin, ceftriaxone, cefepime, imipenem, tigecycline, piperacillin + tazobactam (tazocin), vancomycin, penicillin, gentamicin, linezolid, chloramphenicol, erythromycin, methicillin, ampicillin, and moxifloxacin were obtained and used as per the manufacturer's instructions.

Data were entered and analyzed using the Statistical Package for Social Sciences (SPSS) v.23.0 (IBM, Armonk, US). Descriptive statistics were applied to find frequencies and percentages. Charts and tables were constructed.

\section{Results}

A total of 186 samples were collected during this study period with 85 (45.7\%) samples from males, 95 (51.1\%) from females, while gender was missing for six (3.2\%) patients. Positive growth was observed in 84 (45.2\%) samples. The gram-negative bacilli contributed a major number of isolates $(75 ; 68.4 \%)$, the remaining nine $(31.6 \%)$ were caused by gram-positive cocci.

Out of the bacteria isolated from positive growth cultures, Acinetobacter $(45 ; 53.6 \%)$ was the most common isolate followed by Klebsiella $(11 ; 13.1 \%)$. There were seven cases $(8.3 \%)$ of Staphylococcus aureus which included one (1.3\%) methicillin-resistant Staphylococcus aureus (MRSA). The least common isolate was Enterococcus (2; 2.4\%) (Figure 1). 


\section{Cureus}

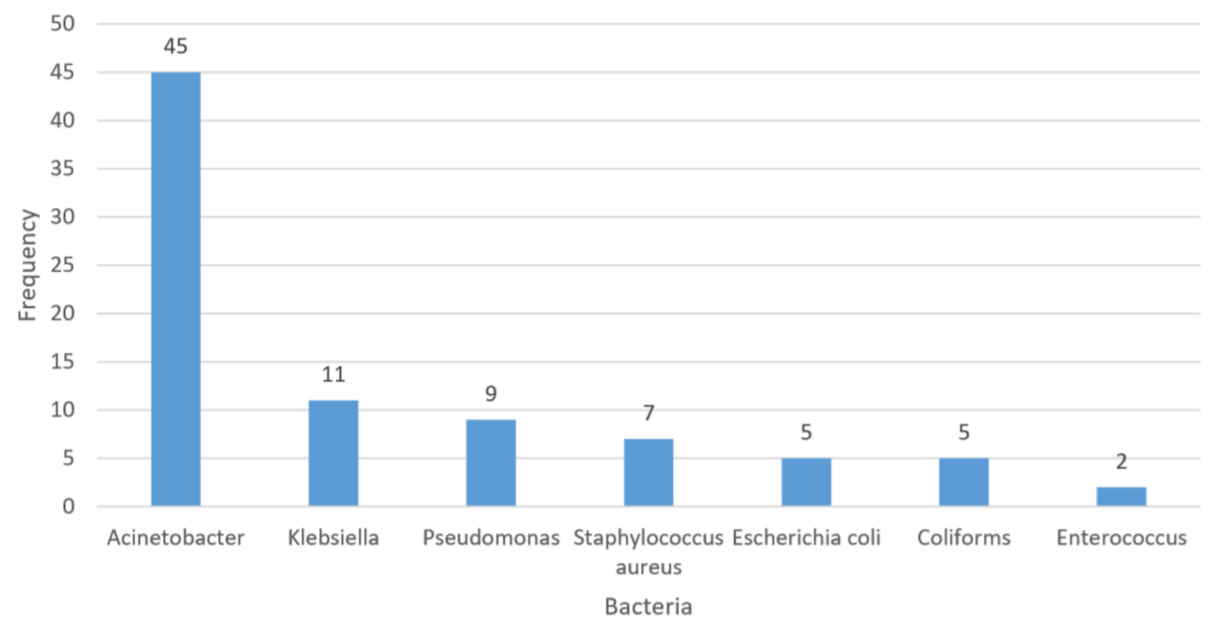

FIGURE 1: Bacterial growth in tracheal secretions $(n=84)$

Acinetobacter was most sensitive to tigecycline (94.7\%) while the gram-positive cocci, i.e., Staphylococcus aureus and Enterococcus showed susceptibility to vancomycin (100\%) (Table 1).

\begin{tabular}{llll} 
Antibiotics & Staphylococcus aureus & Enterococcus \\
Vancomycin & $100 \%$ & $100 \%$ \\
Penicillin & $0 \%$ & - \\
Gentamicin & $100 \%$ & $0 \%$ \\
Linezolid & - & $100 \%$ \\
Chloramphenicol & $100 \%$ & - \\
Erythromycin & $25 \%$ & $0 \%$ \\
Methicillin & $0 \%$ & - \\
Ampicillin & - & $0 \%$ \\
Moxifloxacin & - & $50 \%$ \\
\hline TABLE 1: Antibiotic sensitivity pattern of gram-positive cocci in tracheal secretions
\end{tabular}

The gram-negative bacilli such as Acinetobacter, Klebsiella, and Pseudomonas showed resistance to higher generation cephalosporins such as ceftriaxone and cefepime (100\%). The susceptibility pattern of gram-negative bacilli is shown in Table 2 . 


\section{Cureus}

\begin{tabular}{|c|c|c|c|c|c|}
\hline Antibiotic & Acinetobacter & Klebsiella & Pseudomonas & Escherichia coli & Coliforms \\
\hline Amikacin & $4.7 \%$ & $54.5 \%$ & $22.2 \%$ & $80 \%$ & $80 \%$ \\
\hline Amoxicilllin clavulanate (augmentin) & $0 \%$ & $0 \%$ & - & $0 \%$ & $40 \%$ \\
\hline Aztreonam & - & - & $28.6 \%$ & - & - \\
\hline Ceftazidime & $0 \%$ & $0 \%$ & $20 \%$ & $0 \%$ & - \\
\hline Cefoperazone + sulbactam (sulzone) & $7.7 \%$ & $81.8 \%$ & $66.7 \%$ & $50 \%$ & $60 \%$ \\
\hline Ciprofloxacin & $0 \%$ & $0 \%$ & $22.2 \%$ & $20 \%$ & $50 \%$ \\
\hline Ceftriaxone & $0 \%$ & $0 \%$ & $0 \%$ & $25 \%$ & $60 \%$ \\
\hline Cefepime & $0 \%$ & $0 \%$ & $0 \%$ & $33.3 \%$ & $50 \%$ \\
\hline Imipenem & $2.5 \%$ & $70 \%$ & $28.6 \%$ & $100 \%$ & $40 \%$ \\
\hline Tigecycline & $94.7 \%$ & - & - & - & - \\
\hline Piperacillin + tazobactam (tazocin) & $2.5 \%$ & $71.4 \%$ & $12.5 \%$ & $50 \%$ & $75 \%$ \\
\hline
\end{tabular}

TABLE 2: Antibiotic sensitivity pattern of gram-negative bacilli in tracheal secretions

\section{Discussion}

The resistance to conventional antibiotics is severely increasing in bacteria in clinical and nonclinical settings [10]. The rate of nosocomial infection is also steadily mounting in the patients admitted in the ICU due to excessive invasive procedures performed including artificial ventilator support [11]. This constantly emerging resistance is a serious situation implying the need for new regulations for the cautious use of antibiotics and refining the conditions of hospitals to prevent further exacerbation of resistance shown by the bacteria.

The percentage of samples showing positive growth in our study was $45.2 \%$. In a study conducted by Gupta et al., the percentage of positive growth was 53\% [7]. In another study by Chandra et al., the positive samples were $72.3 \%$ [12]. In a study conducted in the setting of Pakistan by Malik et al., the positive cultures came out to be $83 \%$ [13]. This marked decrease in our study can be attributed to the better infection control measures in the ICU setup of our hospital. However, the convenient sampling technique used in our study might be a limiting factor for the decreased percentage of positive growth.

In our study, gram-negative bacilli were more common causative agents (68.4\%) as compared to gram-positive cocci, which were $31.6 \%$ of the total positive cultures. This was consistent with other researches by Chandra et al., in which the gram-negative bacilli were $85 \%$ and Gupta et al., in which $86 \%$ of the samples were gram-negative bacilli [7,12]. A study by Deepti et al. showed that more of the isolates from the patients were gram-negative enteric aerobic bacteria, with Klebsiella being the most common species followed by Acinetobacter and Pseudomonas [12]. This can be attributed to the fact that the majority of the nosocomial infections are caused by gram-negative bacteria which are more dangerous and difficult to treat. This calls for strict measures against the spread of gram-negative bacilli, especially in the ICU setting. 
In our study, Acinetobacter (53.6\%) was the most common isolate. In a study by Malik et al., conducted in Lahore, Pakistan, the commonest bacterium isolated from tracheal secretions was Klebsiella pneumoniae (35.4\%) [13]. Similarly, in a study by Chandra et al., Klebsiella (32.35\%) was the most common isolate [12]. However, in one study by George et al., Acinetobacter was the most common isolate (37.5\%), followed by Pseudomonas (21.8\%) and Klebsiella (15.6\%) [14]. The rise in Acinetobacter in our study, especially in the ICU setup, can be attributed to the dramatic increase in the occurrence of multi-drug resistant isolates. In addition, this organism has the ability to survive in humid and dry conditions for longer periods, resulting in nosocomial outbreaks $[15,16]$.

The second most common isolate in our study was Klebsiella (13.1\%). In a study conducted by Malik et al., in Pakistan, the most common bacterium isolated from tracheal secretions was Klebsiella pneumoniae (35.4\%) [13]. Another study by Chandra et al. showed Klebsiella (32.35\%) to be the most common isolate [12]. Pseudomonas was the third-most common isolate present in our study. A study by Chandra et al. showed similar results [12].

In our study, Acinetobacter was most sensitive to tigecycline (94.7\%) followed by sulzone (7.7\%). A study by Malik et al. showed 69\% sensitivity of Acinetobacter to sulzone [17]. In another study by Anusha et al., Acinetobacter was most sensitive to imipenem and ciprofloxacin [3]. The decrease in sensitivity, especially to sulzone, can be attributed to the emergence of resistance to the drugs that were conventionally administered.

Our study showed Klebsiella to be most sensitive to sulzone (81.8\%) and tazocin (71.4\%). Pseudomonas was shown to be $66.7 \%$ sensitive to sulzone. In a study by Malik et al., the sensitivity of Klebsiella to sulzone was 62\%, while that of Pseudomonas was $71.2 \%$ [17]. Both Klebsiella and Pseudomonas showed a gradual decrease in the sensitivity to drugs. These gramnegative bacteria have developed resistance to multiple drugs which can be associated with cross-infections and other factors like the unjust use of antibiotics [18].

The other gram-negative bacilli of our study included Escherichia coli and coliforms which were sensitive to amikacin (80\%) and imipenem (100\%). Escherichia coli, in a study by Anusha et al., showed sensitivity to both amikacin and imipenem [3].

The gram-positive cocci, Staphylococcus aureus and Enterococcus, were sensitive to vancomycin (100\%). This was consistent with a study by Gupta et al. which showed no change in antimicrobial resistance patterns [7].

The resistance patterns of bacteria in our study showed Acinetobacter to be $100 \%$ resistant to ceftazidime, ceftriaxone, ciprofloxacin, and cefepime. In the research by Gupta et al., Acinetobacter showed resistance to cephalosporins and aminoglycosides [7]. In another research by Chandra et al., $86 \%$ of the samples showed resistance against ceftriaxone and ceftazidime, each [12]. This depicted a pattern of increasing resistance to the drugs. In our study, out of all the gram-negative bacilli, Acinetobacter showed increasing resistance to carbapenems. Another study in Pakistan by Malik et al. showed similar results [17]. This is an alarming situation as the emergence of multi-drug resistant (MDR) and extensive drug resistant (XDR) pathogens in tracheal secretions is increasing morbidity and mortality in patients, making treatment difficult and expensive.

The remaining gram-negative bacteria in our study displayed resistance to the higher generations of cephalosporins, fluoroquinolones, penicillin, and drugs such as ceftazidime, ceftriaxone, ciprofloxacin, and cefepime. The broad-spectrum antibiotics showed sensitivity to an extent, but there was an alarming rise in the resistance to drugs such as carbapenems. This was in consistency with the pattern shown in the research by Gupta et al. and Malik et al. [7,17] 
The limitation of our research included the decreased time duration of the study. Moreover, the study was carried out in a single tertiary care hospital due to which the generalization of results to the whole population cannot be carried out.

\section{Conclusions}

Gram-negative bacilli were predominant in tracheal secretions with Acinetobacter being the most common species isolated. Tigecycline was found to be effective against Acinetobacter. The resistance to cephalosporins and penicillins is shown to be established by Acinetobacter, Pseudomonas, and Klebsiella. One of the grave concerns related to hospital-acquired infections is the upsurge of multi-drug resistance among the respiratory pathogens which have also extended into the community. There is a need for limited and objective use of antibiotic therapy according to the new guidelines modified under the light of such researches.

\section{Additional Information}

\section{Disclosures}

Human subjects: Consent was obtained by all participants in this study. Institutional Research Forum, Rawalpindi Medical University issued approval RSRS-2018-P-05. This research forum certifies that: 1 . The above-mentioned study is within the ethical boundaries. 2 . There is no duplication of the above-mentioned study in this institution. 3 . The forum acknowledges the importance of the study. 4 . None of the investigator and co-investigator participating in this study took part in the decision making and voting procedure for this study. Animal subjects: All authors have confirmed that this study did not involve animal subjects or tissue. Conflicts of interest: In compliance with the ICMJE uniform disclosure form, all authors declare the following: Payment/services info: All authors have declared that no financial support was received from any organization for the submitted work. Financial relationships: All authors have declared that they have no financial relationships at present or within the previous three years with any organizations that might have an interest in the submitted work. Other relationships: All authors have declared that there are no other relationships or activities that could appear to have influenced the submitted work.

\section{References}

1. Florey H, Carleton HM, Wells AQ: Mucus secretion in the trachea . Brit J Exp Path. 1932, $13: 269$.

2. Yeager Jr H: Tracheobronchial secretions. Am J Med. 1971, 50:493-509. 10.1016/00029343(71)90338-X

3. Anusha N, Madhu KP, Arun BJ, Vidyasagar B: Microbiological profile and sensitivity pattern of endotracheal secretions in mechanically ventilated patients in the ICU. J Evid Based Healthc. 2014, 1:1177-1184.

4. Safdar N, Crnich CJ, Maki DG: Nosocomial infections in the intensive care unit associated with invasive medical devices. Curr Infect Dis Rep. 2001, 3:487-95. 10.1007/s11908-001-00855

5. National Nosocomial Infections Surveillance (NNIS) system report, data summary from Jan 1992-April 2000. Am J Infect Control. 2000, 28:429. 10.1067/mic.2000.110544

6. Fiel S: Guidelines and critical pathways for severe hospital-acquired pneumonia . Chest. 2001, 119:412. 10.1378/chest.119.2_suppl.412S

7. Gupta P, Gupta S, Singh JB: Bacteriological profile and the antibiotic susceptibility pattern of endotracheal secretions In the ICU of a tertiary care hospital. Journal of Evolution of Medical and Dental Sciences. 2018, 7:2210-3. 10.14260/jemds/2018/497

8. Kiran Tandia, J.L. Wadhwani, Manuj Sharma: A clinical study of pattern of microbiological colonization of endotracheal tube aspirate on mechanically ventilated patients. Int J Sci Res. 2015, 4:785-787. https://www.ijsr.net/archive/v4i11/NOV151236.pdf

9. Dautzenberg MJ, Wekesa AN, Gniadkowski M, et al.: The association between colonization 
with carbapenemase-producing enterobacteriaceae

andoverallICUmortality:anobservationalcohort study. Crit Care Med. 2015 Jun, 43:1170.

10.1097\%2FCCM.0000000000001028

10. Kumarasamy KK, Toleman MA, Walsh TR, et al.: Emergence of a new antibiotic resistance mechanism in India, Pakistan, and the UK: a molecular, biological, and epidemiological study. Lancet Infect Dis. 2010, 10:597-602. 10.1016/S1473-3099(10)70143-2

11. Pattanayak C, Patanaik SK, Datta PP, Panda P: A study on antibiotic sensitivity pattern of bacterial isolates in the intensive care unit of a tertiary care hospital in Eastern India. Int J Basic Clin Pharmacol. 2013, 2:153-9. 10.5455/2319-2003.ijbcp20130307

12. Chandra D, Laghawe A, Sadawarte K: Microbiological profile and antimicrobial sensitivity pattern of endotracheal tube aspirates of patients in ICU of a tertiary care hospital in Bhopal, India. Int J Curr Microbiol App Sci. 2017, 6:891-5. 10.20546/ijcmas.2017.603.104

13. Malik M, Malik MI, Sajjad A: Pattern of bacterial pathogens isolated from endotracheal secretions in Intensive care unit (ICU) patients of a tertiary care hospital of Lahore. Pak J Pathol. 2018, 29:46-48.

14. George P, Sequiera A: Antimicrobial sensitivity pattern among organisms which were isolated from the endotracheal aspirates of patients with ventilator associated pneumonia. J Clin Diagn Res. 2010, 4:3397-401. 10.18203/2320-6012.ijrms20175735

15. Baraibar J, Correa H, Mariscal D, Gallego M, Vallés J, Rello J: Risk factors for infection by Acinetobacter baumannii in intubated patients with nosocomial pneumonia. Chest. 1997, 112:1050-4. 10.1378/chest.112.4.1050

16. Paterson DL: The epidemiological profile of infections with multidrug-resistant Pseudomonas aeruginosa and Acinetobacter species. Clin Infect Dis. 2006, 43:43-8. 10.1086/504476

17. Malik MI, Malik M, Chaudhry A: Antimicrobial susceptibility pattern of bacteria Isolated from tracheal secretions in admitted patients of Lahore General Hospital. Pak J Chest Med. 2018, 24:72-77.

18. Kumari HV, Nagarathna S, Chandramuki A: Antimicrobial resistance pattern among aerobic gram-negative bacilli of lower respiratory tract specimens of intensive care unit patients in a neurocentre. Indian J Chest Dis Allied Sci. 2007, 49:19. 